PoS $\quad \begin{aligned} & \text { PROCEEDINGS } \\ & \text { OF SCIENCE }\end{aligned}$

\title{
Mini neutron monitor measurements at the Neumayer III station and on the German research vessel Polarstern
}

\author{
B. Heber* \\ Christian-Albrechts-University Kiel, Germany \\ E-mail: heber@physik.uni-kiel.de \\ D. Galsdorf \\ Christian-Albrechts-University Kiel, Germany

\section{J. Gieseler} \\ Christian-Albrechts-University Kiel, Germany

\section{K. Herbst} \\ Christian-Albrechts-University Kiel, Germany
}

M. Walther

Deutsches Elektronen-Synchrotron DESY, D-15738 Zeuthen

\section{A. Stoess|}

Deutsches Elektronen-Synchrotron DESY, D-15738 Zeuthen

H. Krüger

Center for Space Research, North-West University, Potchefstroom 2520, South Africa

H. Moraal

Center for Space Research, North-West University, Potchefstroom 2520, South Africa

G. Benadé

Center for Space Research, North-West University, Potchefstroom 2520, South Africa 
Neutron monitors (NMs) are ground-based devices to measure the variation of cosmic ray intensities. They are reliable devices but difficult to install because of their size and weight. Therefore a portable mini NM (MNM) that can be installed as an autonomous station at any location that provides suitable conditions has been developed recently. The first continuous measuring MNMs are installed at Neumayer III and the German vessel Polarstern. They are providing scientific data since October 2012 and January 2014, respectively. NM measurements are influenced by the (variable) geomagnetic field and the atmospheric conditions. Thus, in order to interpret the data, a detailed knowledge of the instrument sensitivity with geomagnetic latitude (rigidity) and atmospheric pressure is essential. The rigidity dependence is determined experimentally by utilizing several latitude scans. The Polarstern was specially designed for working in the polar seas and sails usually twice a year in areas with rigidity ranges below $1 \mathrm{GV}$ and above $10 \mathrm{GV}$. The results of different latitude scans from October 2012 to January 2015 will be presented and discussed in the framework of a yield function.

The 34th International Cosmic Ray Conference,

30 July- 6 August, 2015

The Hague, The Netherlands

${ }^{*}$ Speaker. 


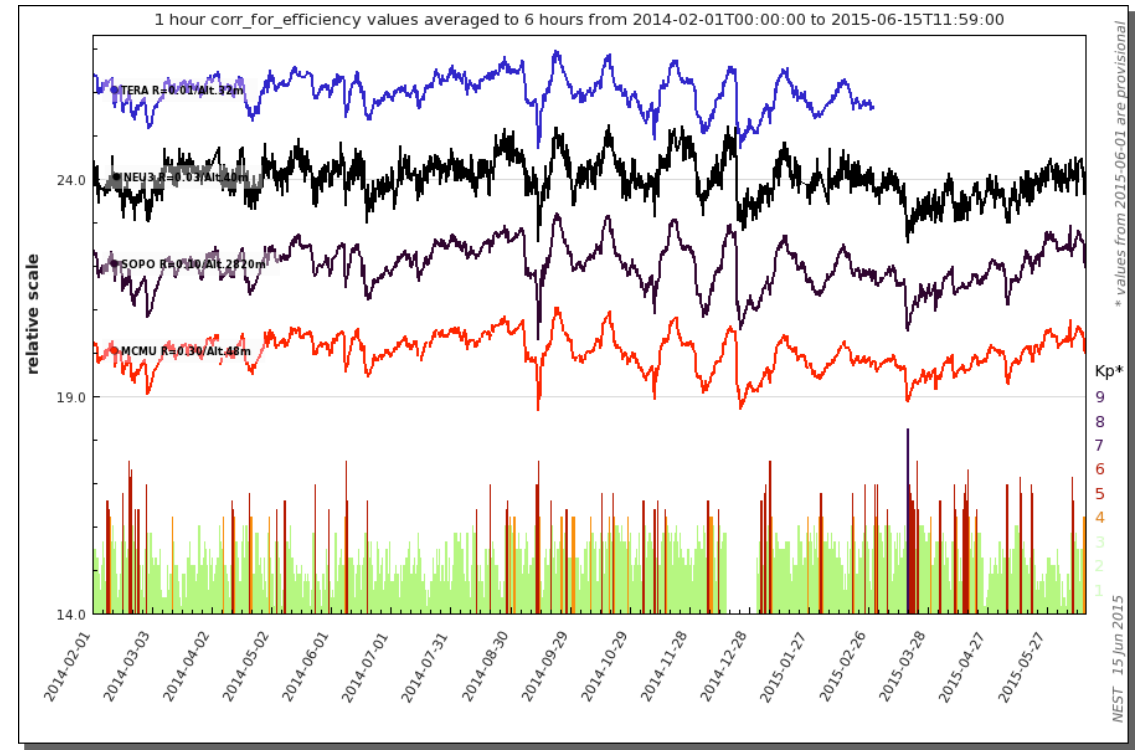

Figure 1: From top to bottom: $1 \mathrm{~h}$ averages of the relative galactic cosmic ray intensity variation as measured by the neutron monitor at Terre Adelie, the mini neutron monitor at the Neumayer III station, the neutron monitor at South Pole and at McMurdo (from nest2.nmdb.eu). In order to indicate disturbed time periods, the $3 \mathrm{~h}$ averaged $\mathrm{K}_{p}$-values are plotted below.

\section{Introduction}

Ground-based measurements of galactic cosmic rays (GCRs) have been performed since their discovery by Viktor Hess in 1912. Since the 1950s neutron monitors (NMs) are utilized for these kind of measurements (Shea and Smart, 2000; Simpson, 2000). Because NMs are integral counters with a threshold energy defined by the minimum of the magnetic cutoff energy and the shielding by the atmosphere, several monitors have been installed world-wide. This should allow to determine the energy spectra of GCRs as well as solar energetic particles (SEPs) during Ground Level Enhancements. In order to extend the existing neutron monitor network, a lightweight mini neutron monitor has been developed (Krüger et al., 2008; Krüger and Moraal, 2013). This device is expected to achieve count rates of 1 count/second at sea level, allowing a statistical accuracy on the percent level by using hourly averages (Krüger and Moraal, 2013; Heber, 2015). The system has been setup as a mobile station that can be placed at almost any location in the world that can provide an office, power supply and internet access. In order to make the data easily accessible, they are provided to the neutron monitor database (NMDB, www.nmdb.eu). One of these devices was successfully installed in February 2014 at the German research station Neumayer III located at the Ekström Shelf Ice, Atka Bay, north-eastern Weddell Sea $\left(70^{\circ} 40^{\prime} \mathrm{S}, 008^{\circ} 16^{\prime} \mathrm{W}\right)$, and another one is mounted on the German research vessel Polarstern (http://www.awi.de/en/infrastructure/ships/polarstern/).

In order to investigate the long term stability, we compare the measurements made at Neumayer III with observations of the neutron monitors at McMurdo, Terre Adelie and on South Pole. These are detectors located in Antarctica and are part of the neutron monitor database consortium. Data can be retrieved by utilizing the tool NEST (nest2.nmdb,eu). Fig. 1 shows the corresponding hourly averaged data from February 2014 to June 2015 from Terre Adelie, Neumayer III, South 

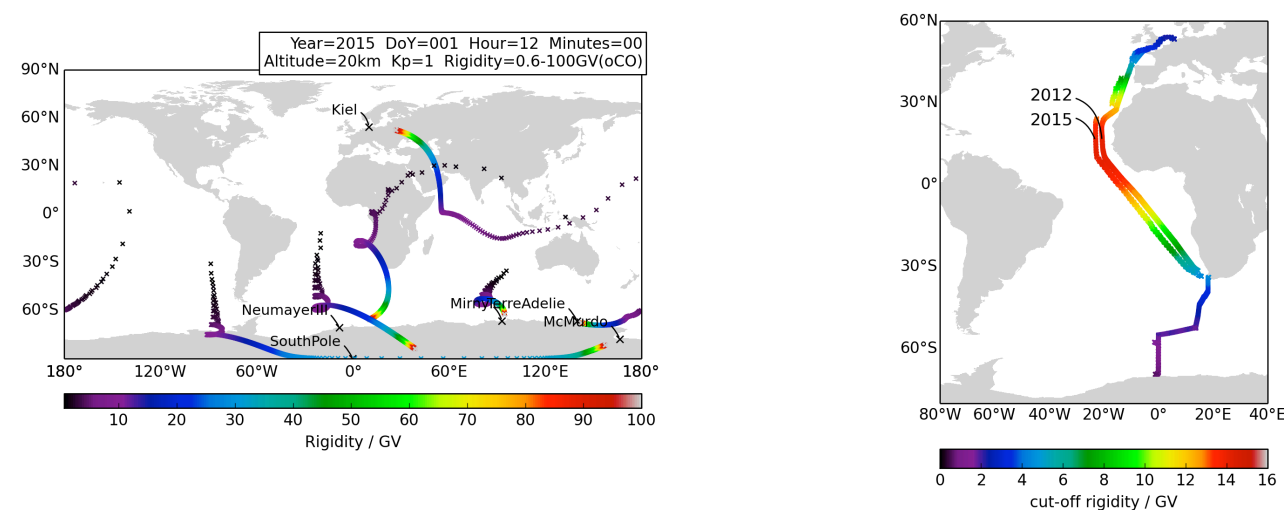

Figure 2: Left: Location and asymptotic directions for the neutron monitor stations at South Pole, Mirny, McMurdo, Terre Adelie, and Neumayer III. The right panel displays the cut-off rigidity of the Polarstern on its way to and from Bremerhaven to Neumayer III.

Pole and McMurdo. The $\mathrm{K}_{p}$-index has been added in order to indicate time periods with high geomagnetic activity. The above analysis shows that the data indeed could be valuable for further studies. Therefore the amplitude of two Forbush decreases that are correlated to the passage of an interplanetary disturbance (Richardson and Cane, 2015) are compared to the amplitude measured by McMurdo. Recently, Mishev et al. (2013) published a set of neutron monitor yield functions. These functions are compared to the measured relative variations that were observed during the two Polarstern latitude scans from 2012/2013 and 2014/2015.

\section{Instrumentation}

The design of the mini NM has been described in detail in Krüger and Moraal (2013). The mini NM has a length of $\sim 80 \mathrm{~cm}$ and a radius of $20 \mathrm{~cm}$. The counter itself has a length of $63 \mathrm{~cm}$ (one third of the standard length of the LND25373 and NM64 counters). It is surrounded by a $2 \mathrm{~cm}$ thick moderator made of paraffin wax, which is surrounded by a $5 \mathrm{~cm}$ lead ring acting as a producer of neutrons. In addition, around the lead ring there is an outer paraffin wax reflector with a thickness of $9.5 \mathrm{~cm}$. In this configuration the mass of the whole unit is estimated to be $220 \mathrm{~kg}$ of which $170 \mathrm{~kg}$ are contributed by the lead and $25 \mathrm{~kg}$ by the paraffin wax. With these dimensions and mass it could be conveniently placed at the Neumayer III station and the Polarstern. The location of the different station as well as the asymptotic directions are shown in the left panel of Fig. 2. The right panel gives the cutoff rigidities along the ship track, ranging from zero to above $14 \mathrm{GV}$ close to the equator.

\section{Long term stability}

In order to investigate the long term stability, we compare the daily averaged variations of McMurdo and South Pole with the ones at Neumayer III. Fig. 3 summarizes in the first panel these measurements from February 2014 to June 2015. The black, red and green curve display 

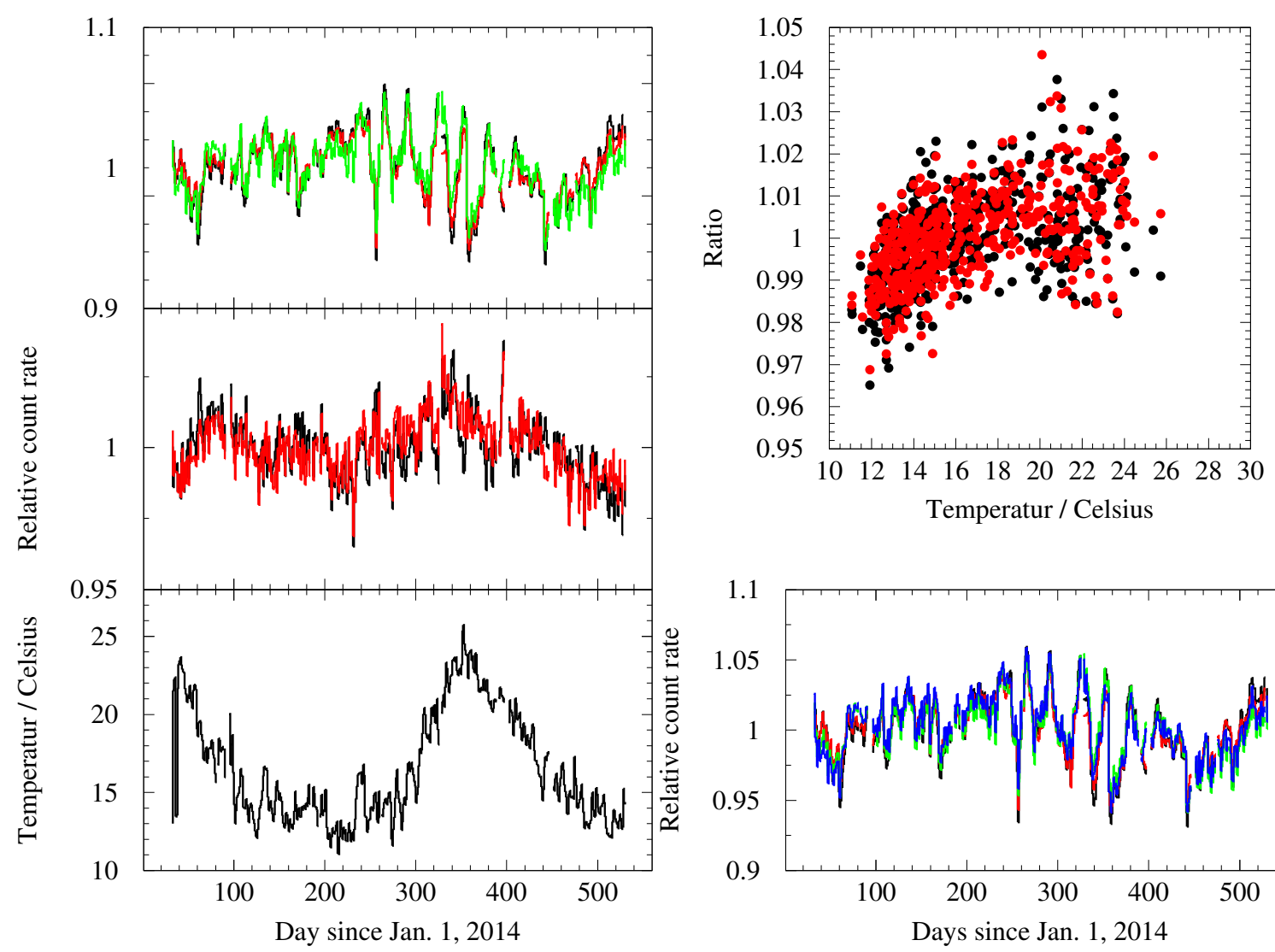

Figure 3: Left from top to bottom: Daily averaged relative variation of the neutron monitors at South Pole (black curve), McMurdo (red curve) and Neumayer III (green curve). The middle panel shows the ratio of the Neumayer III monitor to the one at South Pole (black curve) and at McMurdo (red curve). The lower panel shows the temperature variation at the Neumayer III station. In order to show the correlation between the temperature and the ratios, the upper right panel displays the daily averaged ratios as function of temperature. The temperature corrected time profile of the mini neutron monitor at Neumayer III is given by the blue curve in the lower right panel.

the ones from South Pole, McMurdo and Neumayer III, respectively. Although the statistic is a factor of more than 100 worse for the mini neutron monitor, all three station show similar temporal variations. In order to investigate the difference in more detail, the middle panel show the ratio of the mini neutron monitor at Neumayer III to the one at South Pole (black curve) and McMurdo (red curve). From these curves some systematic trend can be recognized showing larger values in the southern summers than in the corresponding winter time. Since the data used here are only corrected for pressure and not for temperature (see also Heber, 2015), the temperature variation at Neumayer III is given in the lower panel. The daily averaged temperature in the station varies between about $12^{\circ} \mathrm{C}$ in winter to above $25^{\circ} \mathrm{C}$ in summer. Comparing the panels covering the ratios and the temperature suggests a close correlation between these quantities. Therefore the right panel displays the ratios as a function of temperature. A fit to the data points results in a temperature gradient of $0.15 \% /$ degree for which the daily averaged count rates are corrected for in the lower right panel (blue curve). This may become one drawback for our plans to put these 

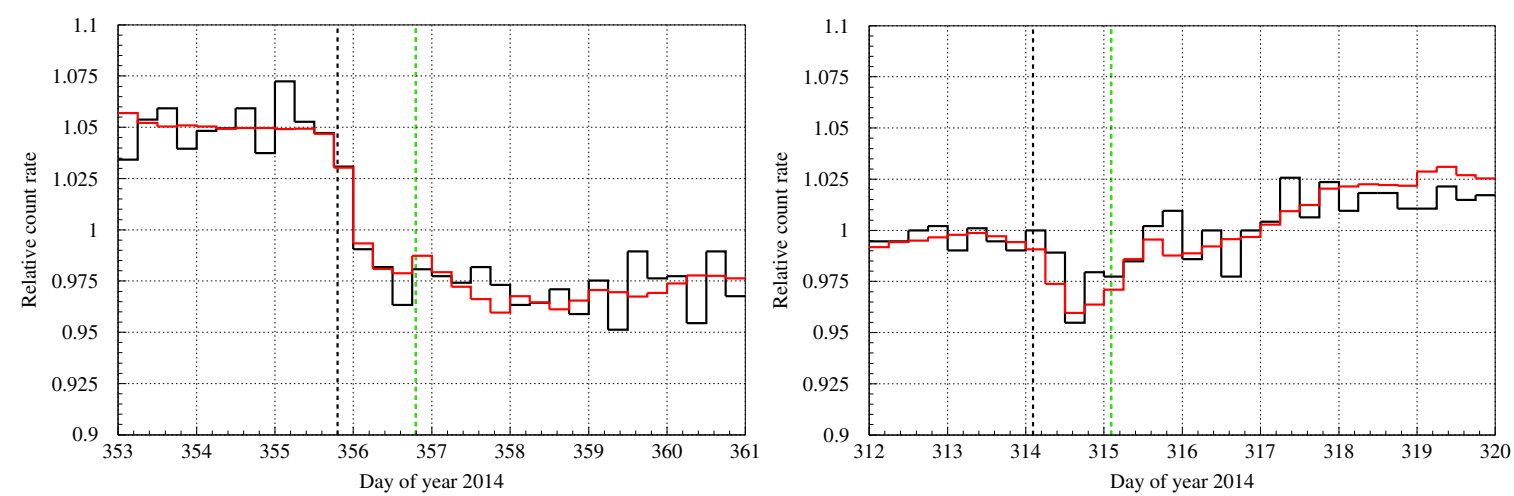

Figure 4: Forbush decreases observed by McMurdo (red curve) and Neumayer III (black curve) associated with the occurrence of a CME. Vertical lines indicate arrival times of the ICME, for details see Richardson and Cane (2015). While there is a significant amplitude of about 7\% for the left event, an amplitude of 3\% for the right event is only significant within $3 \sigma$.

instruments in unattended places. However, only a longer time series would allow us to determine the long-term stability of the mini neutron monitorto a higher precision. The comparison with the other two polar station indicate that a mini neutron monitor shows the same temporal variation and is therefore a valuable instrument in order to expand the existing network.

\section{Sensitivity studies: Forbush decreases}

Forbush (1937) as well as Hess and Demmelmair (1937) were the first to observe short-term intensity decreases using ionization chambers, known as Forbush decreases (FDs). There are two different types of Forbush decreases, one associated with the passage of corotating interaction regions (CIRs, see e.g. Richardson, 2004) and the other with interplanetary coronal mass ejections (ICMEs, see e.g. Cane, 2000; Richardson and Cane, 2011). The largest FDs typically involve ICME that show magnetic cloud (MC) properties (Richardson and Cane, 2011). The amplitude of these variations are still small. Several studies on FDs have been performed utilizing neutron monitors (e.g. Belov et al., 2014, and references therein) which have found amplitudes ranging from more than $10 \%$ to a few \%o. The statistic accuracy of a mini neutron monitor is less than $5 \%$ for an integration period of 6 hours. Thus it should be possible to identify FDs lasting for a day with an amplitude larger than 2\%. Using the ICME list from Richardson and Cane (2015) for 2014, we identified 11 and 8 FDs for McMurdo and Neumayer III, respectively, that were associated to a passage of an ICME. In total 20 ICMEs were listed. Thus only about $50 \%$ were causing a significant FD. Two of them are displayed in Fig. 4. The amplitudes of about $7 \%$ observed during the FD on doy 355 (December, 21) is correlated with an ICME crossing at 19:13 UT. Both, the temporal variation at McMurdo and Neumayer III are in good agreement. The FD shown in the right panel of Fig. 4 occurred on day 314 (November, 10) has an amplitude of about 3\%. Although the statistical significance of the time profile is low for Neumayer III, the agreement between both station is remarkable. Thus we can conclude that for a majority of ICME-caused FDs a network of mini neutron monitors would be a valuable device to extend the existing neutron monitor database. 

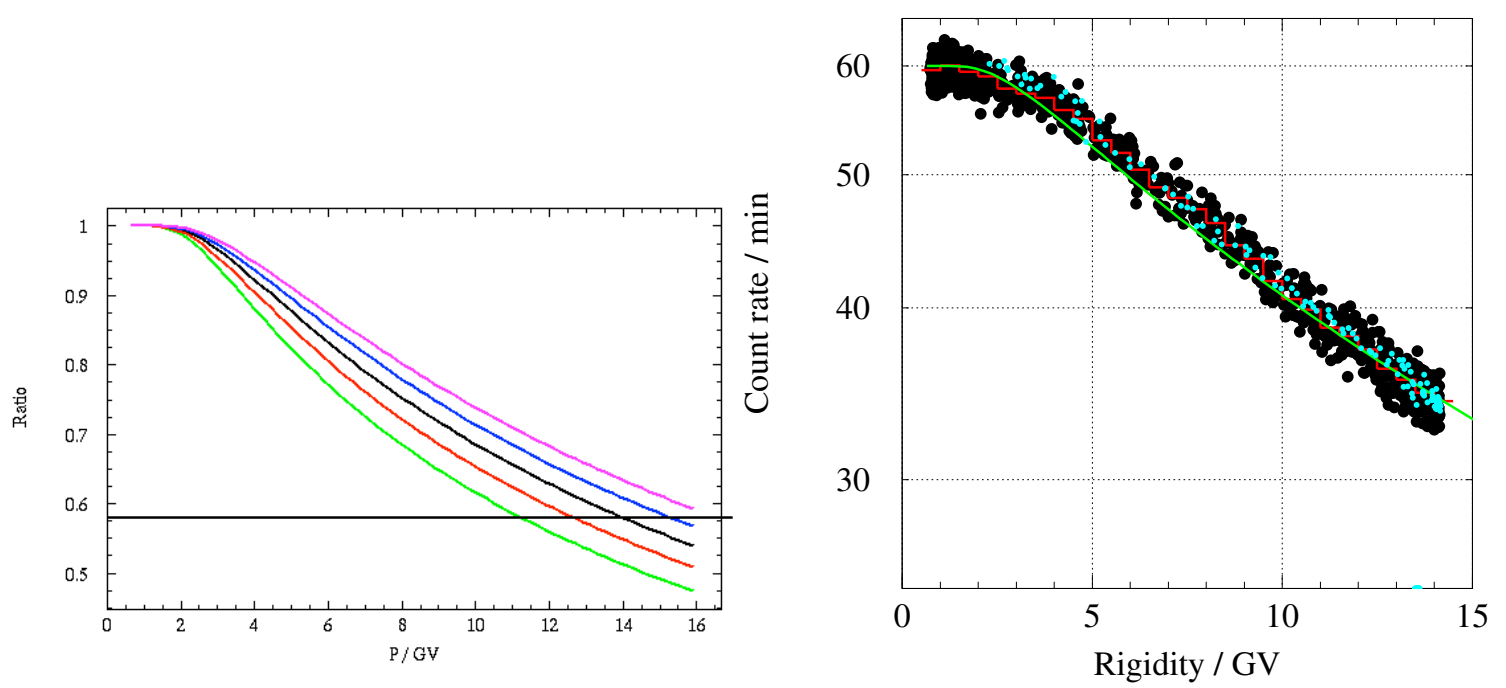

Figure 5: Left: Model results of latitude surveys for different modulation potential varying from 400 to 1200 MV. The right panel displays the measured count rate variation during the 2012/2013 (black dots, and red line) and the 2015 (blue dots) latitude surveys. The green line represent the model results for a modulation potential of $570 \mathrm{MV}$. Note that all count rates and the model results are normalized to each other for rigidities below $1 \mathrm{GV}$.

\section{Latitude surveys of the Polarstern}

Latitudinal surveys have been used in the past to determine the differential response function of a neutron monitor (see e.g. Caballero-Lopez and Moraal, 2012). The intensity of GCRs entering the atmosphere strongly depends on the cut-off rigidity, and with it on the geomagnetic location of the NM. A charged particle, with momentum $p$ and charge $q$, interacting with the Earth's magnetic field $\vec{B}$ has a rigidity $R$ defined as $R=p / q=r_{L} B$, with $r_{L}$ representing the Larmor radius. The cut-off rigidity $R_{C}$ is the rigidity a particle must have in order to reach a certain geographical location. In order to compute $R_{C}$ in an arbitrary magnetic field, numerical computations are mandatory (Herbst et al., 2013; Pilchowski et al., 2010). For our studies we use the simulation code PLANETOCOSMICS (Desorgher et al., 2006). The simulations were carried out for the IGRF model as well as for a magnetic field perturbed by the solar wind according to the Tsyganenko89 model (Tsyganenko, 1989). Figure 2 right displays the result of these calculations along the route of the Polarstern from October 2012 to February 2013. The values range from less than $1 \mathrm{GV}$ at Neumayer III to more than $14 \mathrm{GV}$ in equatorial regions (see Galsdorf, 2014).

Figure 5 displays in its left panel the calculated count rate variation of a neutron monitor using the force field solution from Usoskin et al. (2011) and the neutron monitor yield function calculated by Mishev et al. (2013). The different lines are ordered by the modulation potential ranging from $400 \mathrm{MV}$ (upper curve) to $1200 \mathrm{MV}$ (lower curve). In the right panel of Fig. 5 the prediction for a modulation potential of $570 \mathrm{MV}$ (green curve) is compared with the observations from the 2012/2013 (black dots and red line) and the 2015 latitude survey (blue dots). Note that measurements and model results are normalized to each other at rigidities below $1 \mathrm{GV}$. While the overall amplitude is very well represented by the model there are some details that need to be investigated. 


\section{Summary}

Two modern mini neutron monitors were successfully installed, one in February 2014 at the German research station Neumayer III located at the Ekström Shelf Ice, Atka Bay, north-eastern Weddell Sea $\left(70^{\circ} 40^{\prime} \mathrm{S}, 008^{\circ} 16^{\prime} \mathrm{W}\right)$ and another one on the German research vessel Polarstern (http://www.awi.de/en/infrastructure/ships/polarstern/). Our comparision with a standard neutron monitor shows that the device measured the same features over the last years and can be used for the analysis of FDs that have an amplitude larger than about 3\%. In addition the yield function of Mishev et al. (2013) is capable of describing the amplitude of the measured cosmic ray variation during the two latitude surveys of the Polarstern in 2012/2013 and in 2015.

\section{Acknowledgements}

We acknowledge the NMDB database (www.nmdb.eu), founded under the European Union's FP7 programme (contract no. 213007) for providing data. The data from McMurdo were provided by the University of Delaware with support from the U.S. National Science Foundation under grant ANT-0739620. Kerguelen and/or Terre Adelie neutron monitor data were kindly provided by the French Polar Institute (IPEV, Brest) and by Paris Observatory.

\section{References}

Belov, A., Abunin, A., Abunina, M., Eroshenko, E., Oleneva, V., Yanke, V., Papaioannou, A., Mavromichalaki, H., Gopalswamy, N., and Yashiro, S.: 2014, Solar Physics 289(1), 3949-3960.

Caballero-Lopez, R. A., and Moraal, H.: 2012, Journal of Geophysical Research (Space Physics) 117(A), 12103.

Cane, H. V.: 2000, Space Science Reviews 93(1), 55-77.

Desorgher, L., Flückiger, E. O., and Gurtner, M.: 2006, 36th COSPAR Scientific Assembly. Held 16 - 23 July 2006 36, 2361.

Forbush, S. E.: 1937, Physical Review 51(1), 1108-1109.

Galsdorf, D.: 2014, Cutoff-Steifugkeiten und Asymptotische Richtungen sowie ihre Parameter, $\mathrm{PhD}$ thesis, Christian-Albrechts-Universität zu Kiel.

Heber, B.: 2015, Journal of Physics Conference Series .

Herbst, K., Kopp, A., and Heber, B.: 2013, Annales Geophysicae 31(1), 1637-1643.

Hess, V. F., and Demmelmair, A.: 1937, Nature 140(3), 316-317.

Krüger, H., and Moraal, H.: 2013, Journal of Physics: Conference Series 409(1), 2171.

Krüger, H., Moraal, H., Bieber, J. W., Clem, J. M., Evenson, P. A., Pyle, K. R., Duldig, M. L., and Humble, J. E.: 2008, Journal of Geophysical Research (Space Physics) 113(A), 8101. 
Mishev, A. L., Usoskin, I. G., and Kovaltsov, G. A.: 2013, Journal of Geophysical Research (Space Physics) 118, 2783-2788.

Pilchowski, J., Kopp, A., Herbst, K., and Heber, B.: 2010, Astrophysics and Space Sciences Transactions 6(1), 9-17.

Richardson, I., and Cane, H.: 2015, Near-earth interplanetary coronal mass ejections since january 1996.

URL: $h t t p: / / w w w . s r l . c a l t e c h . e d u / A C E / A S C / D A T A / l e v e l 3 / i c m e t a b l e 2 . h t m$

Richardson, I. G.: 2004, Space Science Reviews 111(3), 267-376.

Richardson, I. G., and Cane, H. V.: 2011, Solar Physics 270(2), 609-627.

Shea, M. A., and Smart, D. F.: 2000, Space Science Reviews 93(1), 229-262.

Simpson, J. A.: 2000, Space Science Reviews 93(1), 11-32.

Tsyganenko, N. A.: 1989, Planetary and Space Science (ISSN 0032-0633) 37, 5-20.

Usoskin, I. G., Bazilevskaya, G. A., and Kovaltsov, G. A.: 2011, Journal of Geophysical Research 116(A), 2104. 